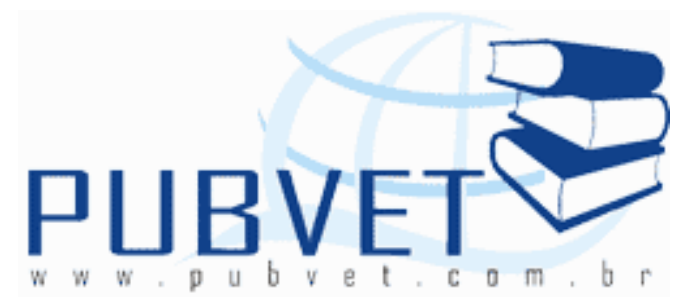

PUBVET, Publicações em Medicina Veterinária e Zootecnia.

\title{
Terminologia anatômica \\ Comparação entre a nomenclatura anatômica humana e a veterinária
}

\author{
Carlos Eduardo Oliveira Sestari ${ }^{1}$, Aline Francielle Corrêa ${ }^{2}$, Antonio Campanha \\ Martinez ${ }^{3}$, Fabrício Singaretti de Oliveira3*
}

1 Acadêmico do Curso de Medicina Veterinária da Universidade Estadual de Maringá - UEM.

2 Acadêmica do Curso de Medicina Veterinária da Universidade estadual de Maringá - UEM.

3* Professor Doutor do Departamento de Medicina Veterinária do Centro de Ciências Agrárias da Universidade Estadual de Maringá-UEM singaretti@ig.com.br

\section{Resumo}

A terminologia anatômica, humana ou veterinária, indica a maneira pela qual devemos nos referir a uma estrutura anatômica para designá-la corretamente. Deve ser utilizada no meio científico e profissional para uniformidade da linguagem e possuir clareza em seu significado. Este trabalha compara a nomenclatura anatômica humana e veterinária, citando os pontos em comum e diferentes entre ambas.

Palavras-chave: nomenclatura, anatomia, terminologia. 


\title{
Anatomical terminology - comparison between human and veterinary anatomical nomenclature
}

\begin{abstract}
The human or veterinary anatomical terminology indicates the way we must refer to an anatomical structure to designate it correctly. It should be used in scientific and professional environmental to provide uniformity of language and cause clarity in its meaning. This paper compares human and veterinary anatomical nomenclature, citing the common and different subjects between them.
\end{abstract}

Keywords: nomenclature, anatomy, terminology.

\section{INTRODUÇÃO}

Os relatos anatômicos humanos iniciaram-se com o surgimento dos próprios hominídeos e ainda hoje se encontram na França e na Alemanha desenhos representativos dos homens e animais dessa época e das suas caçadas. Esta anatomia inicial tinha grande representação de atos místicos e da melhor maneira de se produzir ferimentos em animais durante as caçadas grupais para a obtenção de "carne" para o clã. Esse período compreende uma época de aproximadamente vinte e oito mil anos (SINGER, 1996). No Brasil, essas figuras rupestres encontradas desenhadas com óxido de cobre, em grutas do Nordeste, datam de nove há vinte e três mil anos (BUSETTI \& BUSETTI, 2005).

Com o intuito de facilitar aos padres as confissões de indígenas brasileiros, no início da colonização do Brasil pelos Portugueses e para auxiliar os índios em sua comunicação, Pero de Castilho, um jesuíta nascido em Vila do Espírito Santo, foi levado a preparar uma lista de nomes de partes do corpo humano. Essa lista, de termos Tupis (na língua nativa dos índios) e portugueses, das estruturas anatômicas, em ordem alfabética, parece ser a primeira Nomina Anatomica publicada no Brasil (BEZERRA et al., 2000). 
Até 1895 não havia acordo geral sobre a nomenclatura da anatomia humana ou veterinária. Muitas estruturas possuíam nomes diferentes em diferentes países e muitas eram denominadas com o nome da pessoa que havia feito sua primeira descrição (ELLENPORT, 1986).

É importante a utilização da terminologia própria em todas as áreas médicas. Trabalhos recentes têm destacado essa importância na oftalmologia (LAMY \& DANTAS, 2008), além da importância geral dessa nomenclatura (BUSETTI \& BUSETTI, 2005).

Anatomistas médicos fazem bastante uso de termos anterior e posterior, superior e inferior, os quais apresentam conotações muito diferentes quando aplicados aos quadrúpedes. Deve-se evitar esses termos, exceto para aplicações específicas à cabeça (DYCE et al., 2004).

Deve-se lembrar que há uma nomenclatura similar, mas específica para aves, a Nomina Anatomica Avium (BAUMEL et al., 1993).

O objetivo desse trabalho é reportar termos anatômicos diferentes para a mesma região anatômica, em relação ao homem e aos mamíferos domésticos.

\section{MATERIAL E MÉTODOS}

Foram utilizadas as últimas edições das Terminologia Anatômica Humana (2001) e veterinária (SCHALLER, 1999) para comparação. Estruturas anatômicas de maior importância clínica (médica e cirúrgica) foram observadas quanto à terminologia e as diferenças ou similaridades reportadas neste trabalho (Tabela 1).

\section{RESULTADOS E DISCUSSÃO}

A tabela 1 ilustra algumas diferenças de termos, de grande importância clínica, entre a terminologia humana e veterinária. 
SESTARI, C.E.O. et al. Terminologia anatômica - Comparação entre a nomenclatura anatômica humana e a veterinária. PUBVET, Londrina, V. 5, N. 22, Ed. 169, Art. 1138, 2011.

Tabela 1. Alguns termos diferentes, para a mesma região anatômica, entre a anatomia humana $(\mathrm{H})$ e veterinária $(\mathrm{V})$.

\begin{tabular}{|c|c|c|}
\hline TERMO EM LATIM & $\begin{array}{l}\text { NOMENCLATURA } \\
\text { HUMANA }\end{array}$ & $\begin{array}{c}\text { NOMENCLATURA } \\
\text { VETERINÁRIA }\end{array}$ \\
\hline $\begin{array}{l}\text { Membrum superius (H); } \\
\text { membrum thoracicum (V) }\end{array}$ & Membro superior & Membro torácico \\
\hline $\begin{array}{l}\text { Membrum inferius }(H) ; \\
\text { membrum pelvinum }(V)\end{array}$ & Membro inferior & Membro pélvico \\
\hline Talus & Talus & Talo \\
\hline $\begin{array}{l}\text { Hallux, digitus primus }(H) ; \\
\text { digitus } I(V), \text { pollex* }(V)\end{array}$ & Hálux & Digito I \\
\hline Anterior $(H)$; cranialis $(V)$ & Anterior & Cranial \\
\hline Posterior (H); caudalis (V) & Posterior & Caudal \\
\hline $\begin{array}{l}\text { Articulatio coxofemoralis } \\
(\mathrm{H}) \text {; articulatio coxae }\end{array}$ & Articulação do quadril & $\begin{array}{l}\text { Articulação coxal (ou do } \\
\text { quadril) }\end{array}$ \\
\hline Foramen obturatum & Forame obturado & Forame obturador \\
\hline Abdomen & Abdome & Abdômen \\
\hline Liquor cerebrospinalis & Líquido cerebrospinal & Líquido cefalorraquidiano \\
\hline Nodus sinuatrialis & Nó sinotrial & Nodo sinoatrial \\
\hline Nodus atrioventricularis & Nó atrioventricular & Nodo atrioventricular \\
\hline $\begin{array}{l}\text { Nodus mandibulare }(H) ; \\
\text { lymphonodus mandibulare } \\
\text { (V) }\end{array}$ & Linfonodo submandibular & Linfonodo mandibular \\
\hline \multirow{2}{*}{$\begin{array}{l}\text { Glandula suprarenalis }(H) ; \\
\text { glandula adrenalis (V); } \\
\text { glandula suprarenalis* }(V)\end{array}$} & Glândula supra-renal & Glândula adrenal \\
\hline & Gl. Sudorífera & Gl. Sudorípara \\
\hline $\begin{array}{l}\text { Hypophysis; } \\
\text { pituitaria* (V) }\end{array}$ & Hipófise & $\begin{array}{l}\text { Hipófise ou } \\
\text { pituitária }\end{array}$ \\
\hline Cervix uteri & Colo do útero & Cérvix ou colo do útero \\
\hline Papilla vallata & Papila circunvalada & Papila valada \\
\hline
\end{tabular}

Fonte: TERMINOLOGIA ANATÔMICA (2001) (H); SCHALLER (1999) (V);

Os membros, pelo posicionamento do animal (quadrúpede) ser muito diferente do humano (bípede) (DYCE et al., 2004), talvez sejam os termos que mais apresentam diferenças em sua terminologia (torácico e pélvico para os animais e superior e inferior para os humanos). Ainda devido à essa diferença de posicionamento, em humanos, o termo glândula supra-renal torna-se mais apropriado que o termo adrenal dos animais, pois esta localiza-se acima do rim 
SESTARI, C.E.O. et al. Terminologia anatômica - Comparação entre a nomenclatura anatômica humana e a veterinária. PUBVET, Londrina, V. 5, N. 22, Ed. 169, Art. 1138, 2011.

do homem (MOORE, 1994) e não próxima ao mesmo, como nos animais (FRANDSON et al., 2005).

Termos os mais curtos e simples possíveis seguem um dos princípios da nomenclatura anatômica (ELLENPORT, 1986). Assim, o termo abdome, utilizado pela nomenclatura anatômica humana parece ser mais adequado que o termo abdômen, sugerido pela nomenclatura veterinária, assim como nó, ao invés de nodo sinoatrial ou atrioventricular, forame obturado ao invés de obturador e líquido cerebrospinal ao invés de cefalorraquidiano. Diferentemente, a nomenclatura veterinária parece estar mais de acordo com esse princípio nos termos linfonodo mandibular, contra submandibular, papila valada ao invés de circunvalada, e articulação coxal ao invés de articulação do quadril, utilizados em humanos.

\section{CONCLUSÃO}

Embora semelhantes, as nomenclaturas anatômicas humana e veterinária possuem alguns termos diferentes para designar a mesma estrutura. A nomenclatura humana é mais concisa e simples em alguns termos e a nomenclatura veterinária o é em outros.

\section{REFERÊNCIAS}

BAUMEL,J.J., King, A.S.; Breazile, J.E.; Evans, H.E. \& Vanden Berge, J.C Handbook of avian anatomy: Nomina Anatomica Avium. 2.ed. Nuttall Ornithological Club 23, Cambridge, 779 p. 1993.

BEZERRA, A.J.C., BEZERRA, R.F.A., DI DIO. L.J.A. Brasil 500 anos. Nomenclatura anatômica de um jesuíta no tempo do Descobrimento. Rev Ass Med Brasil. 46(2), 186-90, 2000.

BUSETTI, J.H., BUSETTI, M.P. A nomenclatura anatômica e sua importância. Arq Med ABC. 30(2),119-20, 2005;

DYCE, K.M., SACK, W.O. \& WENSING, C.J.G. Tratado de Anatomia Veterinária. 2.ed. Rio de Janeiro: Elsevier. 2004. p.2.

ELLENPORT, C.R. Introdução Geral. In: Sisson S \& Grossman J.D. (Eds). Anatomia dos Animais Domésticos. 5.ed. Rio de Janeiro: Guanabara Koogan. 1986. v.1, p.2. 
SESTARI, C.E.O. et al. Terminologia anatômica - Comparação entre a nomenclatura anatômica humana e a veterinária. PUBVET, Londrina, V. 5, N. 22, Ed. 169, Art. 1138, 2011.

FRANDSON, R.D.; WILKE, W.L.; FAILS, A.D. Anatomia e Fisiologia dos Animais de Fazenda. Rio de Janeiro: Guanabara Koogan, 2005, p.197.

LAMY, R.; DANTAS, A.M. Nomenclatura anatômica em oftalmologia. Arq Bras Oftalmol.71(3), 446$58,2008$.

MOORE, K.L. Anatomia orientada para a clínica. 3.ed. Rio de Janeiro: Guanabara Koogan.p.198.

SCHALLER, O. Nomenclatura Anatômica Veterinária Ilustrada. São Paulo: Manole. 1999. 614p.

SINGER, C. Uma breve história da anatomia e fsiologia desde os gregos até Harvey. Campinas, Editora Unicamp, 1996. 234p.

TERMINOLOGIA ANATÔMICA. São Paulo: Manole. 2001. 248p. 